\title{
EDUCAÇÃO EM SAÚDE PARA GESTANTES: EXPERIÊNCIA DA IMPLEMENTAÇÃO DE UM GRUPO DE GESTANTES
}

\author{
HEALTH EDUCATION FOR PREGNANT WOMEN: EXPERIENCE OF \\ THE IMPLEMENTATION OF A GROUP OF PREGNANT WOMEN
}

Huanna Raíssa de Medeiros Fernandes ${ }^{1}$

RESUMO: Objetiva-se relatar a experiência da implementação de grupo de gestantes na Unidade Básica de Saúde da Família Irmã Ana Dias, no bairro João XXIII, localizado no município de Caicó - RN. Trata-se de um estudo descritivo, tipo relato de experiência, desenvolvido pelos profissionais da Residência Multiprofissional em Atenção Básica mediante a efetivação e atuação em um grupo de gestantes. Os encontros são realizados quinzenalmente há cerca de 04 meses, têm 01 hora de duração e contam com a participação de em média 08 gestantes. Ressalta- se que todas as gestantes que realizam as consultas de pré-natal são convidadas para os encontros, tanto durante as consultas, como pelos Agentes Comunitários de Saúde. Os temas a serem discutidos são escolhidos pelas gestantes e envolvem temas relativos à gestação, parto, puerpério e cuidados com recém-nascido. A participação dos residentes é em conjunto com os profissionais da Estratégia Saúde da Família, de forma que todos possam contribuir e fazer parte destes momentos. Diante das experiências vivenciadas, verifica-se que alguns temas as gestantes apresentam um pouco mais de insegurança e outros demonstram um vasto conhecimento e autonomia. Além disso, é compreendido que os momentos pontuais de consultas de pré-natal, muitas vezes, não são suficientes para que todas as dúvidas e inquietações sejam sanadas e desmistificadas. Assim, percebe-se que os encontros do grupo consistem em uma importante ferramenta de educação em saúde, quando proporcionam espaços de fortalecimento de vínculo, trocas de conhecimentos e empoderamento das mulheres diante do momento no qual estão vivenciando.

Palavras chave: Educação em Saúde. Gestantes. Atenção Básica.

\footnotetext{
${ }^{1}$ Enfermeira graduada pela Universidade do Estado do Rio Grande do Norte (UERN) e Residente Multiprofissional em Atenção Básica da Escola Multicampi de Ciências Médicas da Universidade Federal do Rio Grande do Norte (EMCM/ UFRN).
} 
ABSTRACT: The objective of this paper is to report the implementation of a group of expectant mothers at the Sister Ana Dias' Basic Healthcare Unity at João XXIII quarter, located in the city of Caico - RN. It is a descriptive study, an experience report developed by the professionals of the Multiprofessional Residency in Basic Attention through the effecting of and proceeding in a group of expectant mothers. The meetings have been held biweekly since 04 months ago, and lasted 01 hour each. They count with the participation of about 08 expectant mothers. We emphasize that all the expectant mothers who take the prenatal care are invited to the meetings during the consultation, as well as by the Community Healthcare Agents. The discussed themes are chosen by the expectant mothers and include themes related to gestation, birth, postpartum and newborn cares. The participation of residents occurs together with the Family Healthcare Strategy professionals, so that everyone can contribute and be part of these moments. Through the experiences we had, we verify that in some themes the expectant mothers show more insecurity, while in others they demonstrate a vast knowledge and autonomy. In addition, we comprehend that the punctual moments of prenatal care, many times, aren't enough to solve and demystify all doubts and uncertainties. So, we notice that the meetings of the group consist in an important tool of healthcare education, as they provide spaces for bond strengthening, knowledge exchange, and women's empowerment through the moment they are living by.

Keywords: Health Education. Pregnant Women. Primary Care. 


\section{INTRODUÇÃO}

$\mathrm{Na}$ gestação ocorrem variadas transformações na vida da mulher como fisiológicas, psicológicas, culturais e sociais, os quais influenciam a sua condição de saúde. Desse modo, durante esse evento as mulheres podem apresentar diversas necessidades de saúde, que devem compor de instrumentos das consultas de prénatal através do trabalho dos profissionais de saúde (MIRANDA; SILVA; MANDÚ, 2018).

Nesse sentido, o acompanhamento de pré-natal adequado tem o objetivo de garantir a evolução da gravidez, proporcionando o nascimento de um recém-nascido saudável, sem interferir negativamente na saúde da mãe, além de debater questões psicossociais e as intervenções educativas e preventivas. Seu início precoce é o principal indicador da qualidade da assistência às gestantes (BRASIL, 2012).

A Rede Cegonha, criada pela Portaria 1.459 de 24 de junho de 2011, é considerado um plano transformador determinada pelo Ministério da Saúde com o objetivo de regularizar o modelo de assistência ao parto e ao nascimento fundamentado nos conhecimentos da equipe multiprofissional, que garante às mulheres o direito ao planejamento familiar e a humanização da assistência ao prénatal, parto e puerpério, assim como a garantia do nascimento seguro e ao crescimento e desenvolvimento saudável às crianças (BRASIL, 2011).

A discussão dos aspectos sociais, subjetivos e físicos do evento reprodutivo e do cruzamento entre eles, constitui um objetivo inserido nas Políticas de Saúde brasileiras direcionada às mulheres. Essa conduta corresponde com o princípio da integralidade que determina diversas práticas do Sistema Único de Saúde (SUS) e a reorganização intencionada da atenção à saúde através da Atenção Básica (MIRANDA; SILVA; MANDÚ, 2018).

No entanto, é comum no acompanhamento de pré-natal os profissionais médicos e enfermeiros focarem nos aspectos físicos da gestação, tais como: o controle das mudanças físicas da gravidez, o surgimento de uma provável 
intercorrência médica e/ ou obstétrica, acompanhamento do crescimento e vitalidade do feto, como também a realização das ações recomendadas. $E$ mesmo da existência de muitos estudos sobre a importância do pré-natal para a saúde da gestante e bebê, essa assistência é ineficiente devido não proporcionar autonomia para a gestante e seu recém-nascido nessa fase (MIRANDA; SILVA; MANDÚ, 2018; SOUZA; BASSLER; TAVEIRA, 2019).

A gravidez é um período intensivo não somente para a gestante, como também para todos os seus familiares e pessoas envolvidas nesse momento. $E$ nesse processo envolve diversos sentimentos e o aumento do interesse dessas pessoas aos ensinamentos e conhecimentos referentes a essa fase e, com isso, esse período se torna oportuno para empregar as práticas de educação em saúde, além de prevenir as preocupações da gestante com os sinais da gravidez, como também que seja capaz de vivenciar essa fase com mais segurança (SOUZA; BASSLER; TAVEIRA, 2019).

Nesse contexto, a educação em saúde é uma intervenção importante para a promoção da saúde no período gestacional com o intuito de diminuir as consequências negativas da insegurança e das preocupações decorrentes dessa fase, visto que muitas vezes as gestantes se encontram em um estado de vulnerabilidade normalmente associada com o recebimento de informações duvidosas (LIMA et al, 2019).

Nesse sentido, no Caderno de Atenção Básica $n^{\circ} 32$, direcionada ao prénatal, recomenda aos profissionais de saúde da Atenção Básica a prática de ações de educação em saúde para as gestantes e seus acompanhantes seja através de grupos específicos, salas de espera, comunidades, escolas e outros locais de troca de experiências com o objetivo de promover autonomia e autocuidado. Este Caderno também sugere temas para serem abordados nas atividades educativas (BRASIL, 2012).

Tornam-se necessárias as intervenções dirigidas às gestantes, na Atenção Básica, sejam elaboradas com o intuito de promover à saúde, contribuir no autocuidado e objetivando o aumento da qualidade de vida. Dessa forma, é essencial que os profissionais de saúde, especialmente os enfermeiros, adotem a aplicação de grupos na atenção à escuta e na discussão de conteúdos de interesse 
das gestantes, contribuindo no sentido de promover saúde durante o período gestacional (SILVA et al, 2017).

Exercer uma assistência de pré-natal de forma integral nem sempre precisa aplicar recursos abundantes para obter os resultados satisfatórios. As atividades educativas podem repercutir no cuidado, proporcionando a evolução do período gestacional e puerperal de forma saudável, resultando na redução da mortalidade materno-infantil (SILVA; AMPARO; SANTOS, 2017).

Diante disso, a gravidez é o período reprodutivo da mulher em que ocorre diversas transformações na vida da gestante e de sua família e é marcado pela insegurança dos sujeitos envolvidos nesse processo. Nesse momento é essencial a realização do acompanhamento de pré-natal, considerado uma das ações da atenção básica, tornando-se necessária a utilização de atividades educativas para esse público como forma de proporcionar autonomia e maior segurança para vivenciar essa fase.

Este estudo tem como objetivo relatar a experiência da implementação do grupo de gestante na Unidade Básica de Saúde da Família (UBSF) Irmã Ana Dias, no bairro João XXIII, localizado no município de Caicó - Rio Grande do Norte (RN).

\section{MÉTODO}

Trata-se de uma pesquisa descritiva, tipo um relato de experiência da implementação do Grupo de gestantes na UBSF Irmã Ana Dias, no bairro João XXIII, localizado no município de Caicó - RN, Brasil, com gestantes que fazem acompanhamento de pré-natal na referida UBSF.

O município de Caicó possui cerca de 62.709 habitantes, de acordo com o último Censo (IBGE, 2010) e, atualmente possui 24 Unidades Básicas de Saúde da Família e um dos bairros deste município, o bairro João XXIII é localizado na Zona Oeste do referido município, próximo ao Rio Barra Nova e entre os bairros Barra Nova e Paulo VI, com uma população de aproximadamente 4.337 habitantes. Desde a sua fundação, o seu processo de ocupação ocorreu resultante da segregação da 
população mais pobre para distante do centro da cidade e foi realizado de forma desorganizada onde muitas ruas possuem relevos irregulares, com saneamento básico precário e é considerado um local onde há uma grande vulnerabilidade da população (ARAÚJO, 2015).

Ressalta-se que nesta UBSF há a atuação dos profissionais do Programa de Residência Multiprofissional em Atenção Básica da Escola Multicampi de Ciências Médicas da Universidade Federal do Rio Grande do Norte (EMCM/ UFRN).

Assim, a idealização do grupo mencionado neste artigo se deu em virtude de, nas consultas de pré-natal, ter sido observado pela enfermeira residente que as gestantes e puérperas sentiam-se inseguras quanto ao processo gestacional, parto e puerpério e apenas as consultas não estavam sendo suficientes para elucidar as inúmeras dúvidas e insegurança.

Nesta Unidade são acompanhadas no pré-natal 24 gestantes e, em média, 8 delas participam do grupo, todas as usuárias são convidadas para os encontros nas consultas de pré- natal e pelos Agentes Comunitários de Saúde (ACS). Nesses encontros, há a participação da equipe de residentes, composta por: enfermeira, fisioterapeuta, nutricionista, assistente social, psicóloga, farmacêutico, profissional de educação física e cirurgião dentista, além do enfermeiro e os ACS integrantes da equipe da Estratégia Saúde da Família (ESF).

As reuniões do grupo acontecem desde o mês de setembro de 2019, ocorrendo quinzenalmente, com duração de 01 hora, no dia da semana mais oportuno para as gestantes e para os profissionais. O espaço para a realização do grupo, na maioria das vezes, é um pátio amplo localizado na própria UBSF.

Os temas a serem discutidos são sugestões das gestantes e envolvem temas relacionados à gestação, parto, puerpério e cuidados com recém-nascido. A metodologia empregada no desenvolvimento das temáticas busca ser desenvolvida com métodos ativos, que possam incentivar a colaboração de todos. Os materiais utilizados pertencem à própria Unidade ou aos profissionais que auxiliam na execução das reuniões.

Destaca-se que, a produção desta pesquisa, não necessitou submeter ao Comitê de Ética em Pesquisa, devido ser um relato de experiência com a sugestão 
de colaboração baseado na prática de educação em saúde de bons hábitos no período gestacional com as gestantes.

\section{RESULTADOS}

Durante o planejamento, foi decidido como tema do primeiro encontro "Os benefícios do aleitamento materno para a mãe e recém-nascido", pois era uma temática bastante relevante visto que muitas gestantes possuíam dúvidas e havia resistência das puérperas em manter o aleitamento materno e, inicialmente, foi decidido entre a equipe que as atividades do grupo seriam mensais para melhor adesão das usuárias. Nessas reuniões também foi decidido o dia, horário, local e solicitado aos Agentes Comunitários de Saúde (ACS) convidar as gestantes de suas respectivas microáreas.

Preparou-se o ambiente em um salão do prédio da UBSF, colocando-se as cadeiras em círculo e uma mesa com os simuladores dos tipos de mamas, como também um boneco simulando um recém-nascido. Inicialmente foi realizado uma roda de conversa com as gestantes sobre os benefícios do aleitamento materno e, em seguida, realizou-se uma simulação do posicionamento e pegas corretas. No final do encontro, os profissionais debateram sobre a frequência das reuniões mensais e as usuárias optaram por encontros quinzenais, visto que o período gestacional é marcado por muitas transformações tanto fisiológicas, psicológicas e quanto a rotina.

Os demais encontros ocorreram no mesmo local e com temáticas escolhidas pelas usuárias que foram: parto, cuidados com o recém-nascido, direitos durante o ciclo gravídico- puerperal, alimentação saudável, alterações fisiológicas maternas no puerpério e seus aspectos emocionais. Nesses encontros foram realizadas rodas de conversa e simulações, como: as alterações do colo do útero durante o período de dilatação; simulação do trabalho de parto e das terapias não-farmacológicas para alívio da dor do trabalho de parto; banho no recém-nascido com o uso de boneco no qual as gestantes praticaram; simulação dos cuidados com o coto umbilical, além de 
dinâmicas do "mitos e verdades dos sintomas verdadeiros e falsos do trabalho de parto" e uso de imagens representativas do puerpério para as usuárias relatarem suas expectativas e experiências do período do pós-parto.

Em um determinado encontro foi realizada uma visita de vinculação com o Hospital do Seridó, maternidade do município e referência para partos de risco habitual de Caicó-RN e municípios vizinhos, no qual foi firmada uma parceria dos residentes multiprofissionais em atenção básica com os residentes multiprofissionais em materno-infantil, atuantes nessa maternidade. Os residentes em materno-infantil apresentaram o hospital e as rotinas desde a admissão até a alta para as gestantes, as situações que devem procurar a urgência e emergência obstétrica, o funcionamento do banco de leite humano e os processos de doação do leite materno, os direitos das gestantes como o direito ao acompanhante no parto, os primeiros cuidados com o recém-nascido. Em seguida, foi finalizado com um lanche saudável como forma de socialização entre elas e os profissionais de saúde e para que se sintam acolhidas no serviço de saúde onde será realizado o seu parto.

Ao longo dos encontros, as temáticas trabalhadas no grupo de gestantes foram bastante exploradas. As gestantes foram participativas e demonstraram interesse e entusiasmo, expressando suas inseguranças e necessidades durante as discussões, no qual foi essencial no planejamento das atividades educativas.

No decorrer dessas ações, houve uma aproximação entre usuárias e profissionais de saúde, possibilitando os momentos de interação, conhecimento e relaxamento.

$\mathrm{Na}$ discussão do tema "Benefícios do aleitamento materno", percebe-se que as gestantes se sentiam inseguras quanto aos benefícios do aleitamento materno para a mãe e bebê, bem como o posicionamento e pega correta na amamentação, como também era forte a crença delas sobre os mitos que prejudicam o aleitamento. As gestantes multíparas relataram suas experiências pregressas com a lactação e todas participaram da demonstração da posição e pega correta da amamentação com o uso de simuladores.

Foram encontrados resultados semelhantes aos descritos nesta pesquisa no estudo de Sardinha et al (2019), em seu relato de experiência do tema aleitamento materno no grupo de gestantes. Ainda é presente a crença dos mitos que dificultam 
a adesão do aleitamento e muitas delas possuíam insegurança quanto ao tema. Esses mesmos autores relataram que é necessário discutir o tema aleitamento materno, visto que muitas dessas usuárias ainda acreditam nesses mitos, podendo prejudicar a prática da lactação. Portanto, as atividades educativas são importantes e incentivam a troca de conhecimentos entre profissionais e gestantes, contribuindo no incentivo à amamentação e na autonomia dessas mulheres na realização desse ato.

O aleitamento materno é considerado um processo difícil e que requer orientações de forma clara com o intuito de promover tranquilidade e confiança na amamentação, resultando na manutenção do aleitamento materno até o sexto mês de vida do bebê (OLIVEIRA et al, 2019).

No segundo encontro houve discussão sobre "Parto" no qual foi percebido que as gestantes se sentiam inseguras e com receio do parto normal devido às dores presentes nesse tipo de parto. Nesse encontro, as mulheres colaboraram na dinâmica dos mitos e verdades do parto normal, participaram da simulação das terapias não-farmacológicas para alívio da dor do parto e observaram os simuladores do parto normal e alterações do colo do útero durante a dilatação. Os relatos da experiência das multíparas como a demonstração dos simuladores contribuíram para que as primíparas se sentissem mais seguras no processo de trabalho de parto e parto.

Os resultados desta pesquisa são semelhantes com o estudo de Alves et al (2019), no qual em sua pesquisa com gestantes de alto risco, foi relatado que muitas destas se sentiam inseguras quanto à identificação do trabalho de parto e possuíam receio em relação a este tipo de parto.

No estudo de Félix et al (2019), a maioria das gestantes, independentemente da idade e quantidade de filhos, não eram orientadas quanto aos sinais de alerta e trabalho de parto no decorrer das consultas de pré-natal, mas admitam sobre a necessidade de avaliação nas situações de sangramento e suas complicações e, neste mesmo artigo, os autores também destacam para a importância das ações educativas para as gestantes.

Em outro estudo Jardim; Silva e Fonseca (2019), destacam que as gestantes entrevistadas relataram que se sentiam inseguras quanto ao parto e recebiam 
orientações de enfermeiros durante o pré-natal, mas essas informações não proporcionavam o empoderamento delas. Esses mesmos autores discutem que essa problemática possibilita a redução da autonomia da mulher no parto resultando na sua subordinação diante das condutas dos profissionais de saúde e aponta que as atividades educativas para as gestantes devem incentivar o empoderamento nesse grupo.

$\mathrm{Na}$ abordagem do tema "Cuidados com o recém-nascido" foi percebido que as gestantes possuíam insegurança quanto aos cuidados com o recém-nascido em relação ao banho, os cuidados com o coto umbilical, a importância do banho de sol, entre outros. Nessa reunião, as mulheres participaram na discussão e da simulação do banho do recém-nascido e dos cuidados com o coto umbilical. Ao final desse encontro, percebeu-se que as gestantes se sentiram mais seguras quanto aos cuidados com o recém-nascido após a prática da simulação.

Os resultados desta pesquisa foram semelhantes ao estudo de Gomes et al (2015), no qual foram promovidas atividades educativas nas consultas de puericultura em uma unidade de saúde em que foram realizadas ações sobre os cuidados dos recém-nascidos com os familiares. Em seguida, aplicou-se um questionário sobre a temática e foi percebido que eles possuíam dúvidas quanto aos cuidados com o neonato, resultando na dificuldade na adesão de uma rotina saudável antes da participação dessas atividades e após as ações. Muitas dúvidas foram esclarecidas. Esses mesmos autores referem que as intervenções de educação em saúde constituem um método relevante para a diminuição da exposição do recém-nascido aos riscos nos quais podem influenciar na aquisição de doenças e morte.

No estudo de Ribeiro et al (2018), na aplicação de um questionário com as puérperas, percebeu-se que as mesmas responderam de forma incorreta algumas perguntas sobre os cuidados com o recém-nascido e, para isso, torna-se importante a realização de intervenções de educação em saúde para o fortalecimento da atenção integral da mãe e do filho, proporcionando orientações para as gestantes e puérperas com o intuito de prevenir danos à saúde materno- infantil.

$\mathrm{Na}$ abordagem da temática "Direitos no ciclo gravídico-puerperal", as gestantes foram muito participativas na discussão do tema e foi percebido o 
desconhecimento das participantes de seus direitos, sendo estes fundamentais no tocante a evitar possíveis abusos de profissionais de saúde durante o momento de vulnerabilidade dessas mulheres.

O estudo de Aires et al (2015), discute que as dificuldades da aplicação das leis que regem sobre os direitos das parturientes se devem ao pouco conhecimento da maioria dos profissionais de saúde e gestantes sobre esses, o que contribui para a sua não aplicação no cotidiano das maternidades. Para isso, torna-se necessário que as instituições de saúde invistam na educação permanente desses profissionais, como forma de garantir às gestantes e parturientes uma assistência de forma humanizada.

Semelhante a este estudo, Jardim; Silva e Fonseca (2019), relataram que a maioria das gestantes desconheciam seus direitos assistenciais e trabalhistas. Esses mesmos autores discutem que a compreensão de seus direitos proporcionará o seu empoderamento.

No encontro seguinte, foi abordado sobre os "Aspectos físicos e emocionais do puerpério", visto que nessa fase ocorrem alterações hormonais, mudanças corporais e da rotina, as quais podem interferir nas emoções das puérperas. Durante a reunião, as gestantes multíparas relataram suas experiências pregressas do puerpério, como as alterações do corpo, as mudanças psicológicas e que tiveram apoio familiar durante esse período. Em seguida, percebeu-se que as mesmas possuíam dúvidas em relação ao planejamento reprodutivo após o parto e relataram sobre a importância de possuir uma rede de apoio forte como forma de prevenção do blues puerperal e da depressão pós-parto. Nesse sentido, o período puerperal é caracterizado por uma fase em que a mulher se encontra mais vulnerável e, se sentindo apoiada, possibilitará uma experiência desse período de forma tranquila (OLIVEIRA et al, 2019).

Semelhantemente ao estudo de Oliveira et al (2019), as puérperas possuíam dúvidas e não eram orientadas pelos profissionais de saúde sobre as alterações fisiológicas maternas no período do pós-parto. Esses mesmos autores relatam sobre a importância de os profissionais de saúde promoverem atividades educativas e orientações no puerpério imediato para as mulheres sobre as transformações físicas e o motivo destas ocorrerem, objetivando o seu autocuidado. 
Em outro estudo, Demarchi et al (2017), em uma pesquisa com gestantes e puérperas relata que a maternidade é caracterizada pelas transformações quanto físicas, psíquicas, cuidados com o recém-nascido, aumento das tarefas domésticas e os sentimentos em relação ao papel variaram entre uma vivência prazerosa e marcados pela preocupação diante da descoberta da gestação.

No puerpério, as mulheres estão uma situação de vulnerabilidade tanto física quanto psíquica e, para isso, é importante que haja investimento na atenção básica para a promoção de atividades educativas com o intuito de orientar não só a gestante como também toda a sua família a fim de melhorar a aplicação das informações prestadas pela equipe de saúde. Torna-se essencial que esses profissionais estejam habilitados para prestar assistência a esse público de forma humanizada e com segurança, excedendo a abordagem somente nos aspectos fisiológicos (DEMARCHI et al, 2017; OLIVEIRA et al, 2019).

No sexto encontro, realizou-se uma visita de vinculação no Hospital do Seridó, maternidade do município de Caicó-RN e referência para partos de risco habitual. Nessa visita, percebeu-se que as gestantes, especialmente as primíparas, demonstraram entusiasmo e segurança durante a exposição das salas de pré-parto, parto, berçário e banco de leite humano e os relatos dos procedimentos da rotina do hospital, realizado pelos residentes multiprofissionais em materno-infantil. Essa visita foi importante para as gestantes do grupo, visto que com o conhecimento prévio da rotina da maternidade onde será realizado o parto aumenta a segurança durante esse processo.

Semelhante ao resultado deste estudo, Popolli et al (2018), discute que as gestantes pesquisadas adquiriram maior segurança após a visita de vinculação na maternidade no qual motivou na escolha pelo parto vaginal. Esses mesmos autores relatam que essa visita proporciona maior humanização, permitindo o aprendizado, alívio e segurança para essas mulheres.

A Lei 11.634/ 2007 garante às gestantes o direito a visita de vinculação com a maternidade onde receberá assistência no parto e/ ou durante as intercorrências gestacionais no contexto do SUS (BRASIL, 2007).

No sétimo encontro foi abordada a temática de alimentação saudável no período gestacional. Durante o grupo, as gestantes mostraram não só insegurança, 
mas também vontade de aprender sobre o assunto. Nesse sentido, sabe-se que uma alimentação balanceada e o fornecimento adequado de nutrientes no período gestacional se constituem como fatores relevantes, uma vez que é a fase na qual as necessidades nutricionais estão aumentadas, com o intuito de permitir adaptações para o organismo materno, bem como o bom desenvolvimento fetal (BUENO et al, 2016). Em formato de roda de conversa foi discutido temas como a importância da hidratação; alguns alimentos ditos "carregados" como amendoim, peixe e leite de vaca; importância de uma alimentação rica em frutas, legumes e mais próxima do natural possível (o lema "descascar mais e desembalar menos" foi muito incentivado). Curiosidades como alimentos dito saudáveis, têm um potencial maior de formar gases, também foram abordados.

Semelhantemente ao estudo de Moreira et al (2018), as gestantes mostravam um comportamento herdado dos seus familiares (mãe, avó, sogra, tia) e por influenciadores digitais do momento. As falas mostraram que havia uma lógica que determinava o que se poderia fazer parte da dieta na gestação e no pós-parto, dando forma as suas escolhas e hábitos alimentares. Como exemplo, acreditavam que alimentos considerados saudáveis, como frutas e leite, deveriam estar mais presentes na sua alimentação e que deveriam evitar as preparações "mais pesadas" por acreditarem não fazerem mal ao bebê.

\section{CONCLUSÃO}

O relato mostrou a relevância de um grupo de gestante de promoção à saúde, evidenciando um cuidado integral e um olhar ampliado às participantes. O grupo atuou como uma extensão do pré-natal e desenvolveu um trabalho de forma dinâmica, no intuito de abarcar dúvidas que não eram relatadas na consulta individual. Ademais, o compartilhamento de saberes e experiências de outras mães conferiu uma maior segurança e autonomia para as de primeira viagem, bem como corrobora para o fortalecimento do vínculo da residência multiprofissional e Estratégia Saúde da Família com o público-alvo. 


\section{REFERÊNCIAS BIBLIOGRÁFICAS}

AIRES, N.T. et al. Aplicabilidade dos direitos das parturientes: do paradigma à realidade. Saúde (Santa Maria). Santa Maria. v. 41, n. 1, p. 263-270, jan./ jul. 2015.

ALVES, F.L.C. et al. Grupo de gestantes de alto-risco como estratégia de educação em saúde. Revista Gaúcha de Enfermagem. Porto Alegre. v. 40, 2019.

ARAÚJO, H.C. de. Segregação urbana e o reflexo das desigualdades sociais: um estudo sobre o bairro João XXIII na cidade de Caicó-RN. 2015. 69 f. Monografia (Bacharelado em Geografia) - Universidade Federal do Rio Grande do Norte. Centro de Ensino Superior do Seridó. Caicó, RN, 2015.

BRASIL. Lei $n^{\circ} 11.634$, de 27 de dezembro de 2007. Dispõe sobre o direito da gestante ao conhecimento e a vinculação à maternidade onde receberá assistência no âmbito do SUS. Diário Oficial da União. Brasília, DF, v. 1, 2007.

BRASIL. Ministério da Saúde. Secretaria de Atenção à Saúde. Departamento de Atenção Básica. Atenção ao Pré-natal de Baixo Risco. Ministério da Saúde. Secretaria de Atenção à Saúde. Departamento de Atenção Básica. - Brasília: Ministério da Saúde, 2012.

BRASIL. Ministério da Saúde. Gabinete do Ministro. Portaria $n^{\circ}$ 1.459, de 24 de junho de 2011. Institui, no âmbito do Sistema Único de Saúde - SUS - a Rede Cegonha. Diário Oficial da União. Brasília, DF, 2011.

BUENO, A.A. et al. Características da alimentação no período gestacional. LifeStyle Journal. São Paulo. v. 3, n. 2, p. 29-42, 2016.

DEMARCHI, R.F. et al. Percepção de gestantes e puérperas primíparas sobre maternidade. Revista de Enfermagem da UFPE on line. Recife. v. 11, n. 7, p. 2663-2673, jul. 2017.

FÉLIX, H.C.R. et al. Sinais de alerta e de trabalho de parto: conhecimento entre gestantes. Revista Brasileira Saúde Materno-infantil. Recife. v. 19, n. 2, p. 343-349, abr./ jun. 2019.

GOMES, A.L.M. et al. Conhecimentos de familiares sobre os cuidados com recém-nascidos. Revista da Rede de Enfermagem do Nordeste. Fortaleza. v. 12, n. 2, p. 258-265, mar./ abr. 2015.

IBGE - INSTITUTO BRASILEIRO DE GEOGRAFIA E ESTATÍSTICA. Censo demográfico: população no último censo. Rio de Janeiro, 2010. 